\title{
TITLE:
}

\section{Inelastic neutron scattering study of low energy excitations in polymer thin films}

\section{$\operatorname{AUTHOR}(\mathrm{S})$ :}

Inoue, R; Kanaya, T; Nishida, K; Tsukushi, I; Shibata, $\mathrm{K}$

\section{CITATION:}

Inoue, R ...[et al]. Inelastic neutron scattering study of low energy

excitations in polymer thin films. Physical Review Letters 2005, 95(5):

056102.

ISSUE DATE:

2005-07-29

URL:

http://hdl.handle.net/2433/39928

RIGHT:

Copyright 2005 American Physical Society 


\title{
Inelastic Neutron Scattering Study of Low Energy Excitations in Polymer Thin Films
}

\author{
Rintaro Inoue, ${ }^{1}$ Toshiji Kanaya, ${ }^{1, *}$ Koji Nishida, ${ }^{1}$ Itaru Tsukushi, ${ }^{2}$ and Kaoru Shibata ${ }^{3}$ \\ ${ }^{1}$ Institute for Chemical Research, Kyoto University, Uji, Kyoto-fu 611-0011, Japan \\ ${ }^{2}$ Chiba Institute of Technology, Narashino, Chiba-ken 275-0023, Japan \\ ${ }^{3}$ Japan Atomic Energy Research Institute, Tokai, Ibaraki-ken 319-1195, Japan
}

(Received 12 February 2005; published 25 July 2005)

\begin{abstract}
We report inelastic neutron scattering measurements on polystyrene thin films in a glassy state in the $\mathrm{meV}$ region. We found in elastic scattering that the mean square displacement $\left\langle u^{2}\right\rangle$ decreased with film thickness, and hence the corresponding force constant $f$ increased. In inelastic and quasielastic scattering, we observed the so-called boson peak at around $1.5 \mathrm{meV}$ and the picosecond fast process for the first time in thin films, both of which decreased in intensity with film thickness. These results were discussed in terms of the potential hardening due to the confinement of polymer chains and/or the interfacial dead layer.
\end{abstract}

DOI: 10.1103/PhysRevLett.95.056102

Polymer thin films show very interesting but unusual properties which are related to many significant phenomena, such as dewetting, adhesion, surface friction, and so on [1]. Therefore extensive investigations have been performed on thin films from both scientific and industrial points of view [2]. One of the most interesting properties in polymer thin films is the reduction of glass transition temperature $T_{g}$ observed for both polystyrene (PS) thin films supported on Si substrate [3-5] and freestanding PS thin films [6,7]. Another interesting finding on thin films is the reduction of thermal expansivity in the glassy state below about $250 \AA$ [8]. It was pointed out that the reduction is due to the lack of annealing [9], but recent careful measurements [10] have revealed that the reduction of the thermal expansivity is still observed after a long annealing far above $T_{g}$, although the reduction is partially caused by the lack of annealing. It is evident that studies on the dynamics of thin films are essential to reveal the molecular origins of the above-mentioned unusual phenomena. Inelastic neutron scattering measurements on polycarbonate (PC) thin films by Soles et al. [11] and freestanding polystyrene thin films by Frick et al. [12] have provided very significant information on the dynamics in the glassy state as well as near the glass transition, but were limited to the elastic scattering within the corresponding energy resolution $(\delta E \sim 1 \mu \mathrm{eV})$. Recently, Hansen et al. [13] also reported quasielastic neutron scattering studies on alkane monolayers combined with molecular dynamics simulation. In this work, we did directly measure the inelastic and quasielastic scattering from the glassy PS films 400 and $1000 \AA$ thick in the meV region, and succeeded for the first time in observing the boson peak as well as the quasielasticlike fast process in picosecond order in the thin films. On the basis of the elastic, inelastic, and quasielastic neutron scattering results, we will discuss the potential hardening in the PS thin films.

We used PS with molecular weight $M_{w}=2.9 \times 10^{5}$ and molecular weight distribution $M_{w} / M_{n}=1.06$, where $M_{w}$ and $M_{n}$ are the weight average and the number average of
PACS numbers: 68.60. $-\mathrm{p}$, 61.12.Ex, 63.50.+x

the molecular weight, respectively. PS thin films 1000 and $400 \AA$ thick were prepared by spin-coating toluene solutions at $2000 \mathrm{rpm}$ on flat glass plates. The thickness was checked by ellipsometry for every 10 sheets, and the average values were $1004 \pm 22 \AA$ and $402 \pm 8 \AA$. The film was removed from the glass surface onto the water surface, then collected on $\mathrm{Al}$ foil $15 \mu \mathrm{m}$ thick, and then annealed at $413 \mathrm{~K}$ for $12 \mathrm{~h}$ after drying in vacuum at room temperature for $2 \mathrm{~d}$. The inelastic neutron scattering intensity from the thin films was extremely weak, and hence 299 sheets of the films on $\mathrm{Al}$ foils were used for the measurements. They were rolled up and placed into a hollow cylindrical $\mathrm{Al}$ cell $14 \mathrm{~mm}$ in diameter and $45 \mathrm{~mm}$ high, and hence no preferred orientation to the scattering vector. The inelastic neutron scattering measurements were performed with an inverted geometry time of flight (TOF) spectrometer LAM-40 with an energy resolution of about $0.2 \mathrm{meV}$ in KEK, Tsukuba, Japan. The measurements were carried out at $11,80,150$, and $230 \mathrm{~K}$ below the bulk glass transition temperature $T_{g}$ of $373 \mathrm{~K}$. At each temperature the TOF spectrum of the empty can including $299 \mathrm{Al}$ foils was carefully subtracted from that of the thin film after correcting for the counter efficiency, the self-shielding, and the incident neutron spectrum. The PS used in this experiment is a protonated one, and the scattering intensity obtained here is mainly governed by the incoherent scattering.

Figure 1 shows the dynamic scattering laws $S(Q, \omega)$ of the thin film $1000 \AA$ thick at $11,80,150$, and $230 \mathrm{~K}$, which were obtained by summing up 6 spectra from $Q=0.87$ to $2.47 \AA^{-1}$. The average value of $Q$ is $1.71 \AA^{-1}$. The spectra were normalized to the total scattering intensity at the lowest temperature $11 \mathrm{~K}$, which was assumed to be identical for each sample to compare the intensities among the samples. A very broad inelastic peak or shoulder, the socalled boson peak, was observed at around $1.5 \mathrm{meV}$ in addition to the central sharp elastic peak within the energy resolution of the spectrometer $\delta E(=0.2 \mathrm{meV})$. We first analyze the elastic scattering. The $Q$ dependence of inco- 


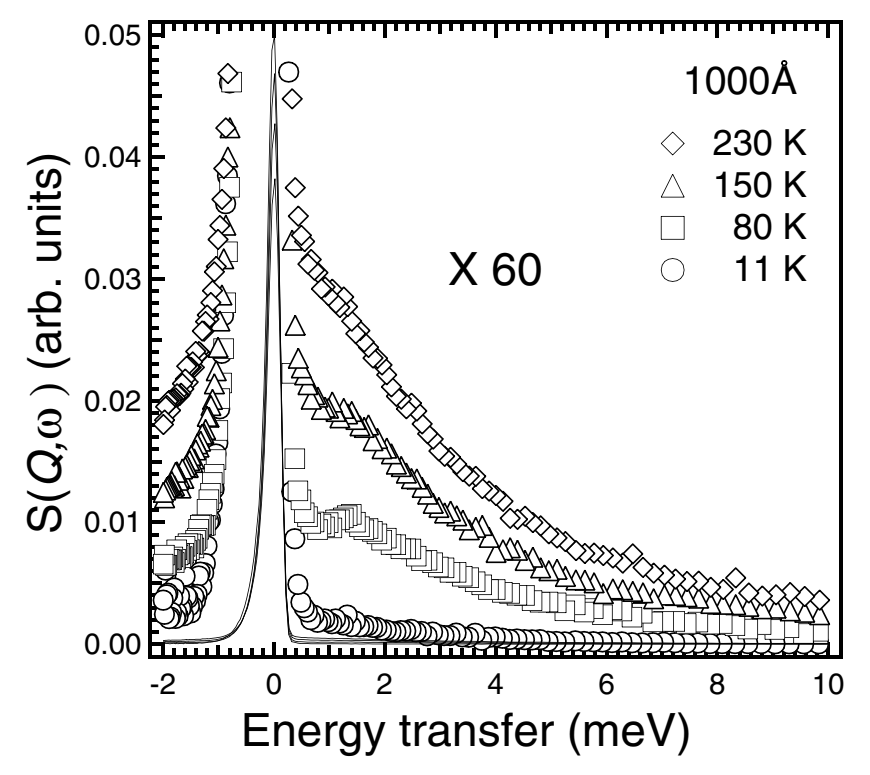

FIG. 1. Dynamic scattering law $S(Q, \omega)$ of $1000 \AA$ PS thin film measured with LAM-40 as a function of temperature. Solid curves show full shapes of the spectra at each temperature and symbols are the expanded ones by a factor 60 .

herent elastic scattering intensity $I_{\mathrm{el}}(Q)$ is related to the mean square displacement $\left\langle u^{2}\right\rangle$ through $I_{\mathrm{el}}(Q) \sim$ $\left[\exp \left(-\left\langle u^{2}\right\rangle Q^{2}\right)\right]$ under the Gaussian approximation. According to this equation, we plotted the logarithm of $I_{\mathrm{el}}(Q)$ against $Q^{2}$ and evaluated $\left\langle u^{2}\right\rangle$ from the slope. In this evaluation, to reduce the effect of coherent scattering, $I_{\mathrm{el}}(Q)$ at a given temperature was divided by $I_{\mathrm{el}}(Q)_{11 \mathrm{~K}}$ at the lowest temperature $11 \mathrm{~K}$. Hence, the evaluated $\left\langle u^{2}\right\rangle$ at the given temperature corresponds to $\left\langle u^{2}\right\rangle_{T}-\left\langle u^{2}\right\rangle_{11 \mathrm{~K}}$. The evaluated $\left\langle u^{2}\right\rangle$ is shown for the bulk and the 1000 and $400 \AA$ thin films as a function of temperature in Fig. 2. The evaluated $\left\langle u^{2}\right\rangle$ decreases with film thickness and this tendency is enhanced with increasing temperature. The $\left\langle u^{2}\right\rangle$ was plotted as a function of film thickness at $230 \mathrm{~K}$ in the inset of Fig. 2, including the result of the $200 \AA$ film [14]. For the $200 \AA$ film, the evaluated $\left\langle u^{2}\right\rangle$ is only onethird of the bulk one. A similar thickness dependence of $\left\langle u^{2}\right\rangle$ has been reported for PC films in the $\mu \mathrm{eV}$ region [11]. It is well known that the mean square displacement $\left\langle u^{2}\right\rangle$ is related to an average force constant $f$ with an assumption of harmonic vibration in the low temperature range (far below $T_{g}$ ) through an equation $\left\langle u^{2}\right\rangle \sim k_{B} T / f$, where $k_{B}$ is the Boltzmann constant. From the slopes in Fig. 2, we evaluated $f$ to be $5.8,6.5$, and $8.7 \mathrm{~N} / \mathrm{m}$ for the bulk and the 1000 and $400 \AA$ thin films, respectively. The value of $f$ increases with decreasing film thickness. By comparing the observed force constants with the theoretically calculated ones for the bulk [15], we found that the obtained $f$ values are close to those for torsion of the $\mathrm{C}-\mathrm{C}$ bond in the main chain and/or the force constant for the interaction potential between nonbonded atoms. Why do the polymers have a higher force constant in the thin films than in the bulk? One

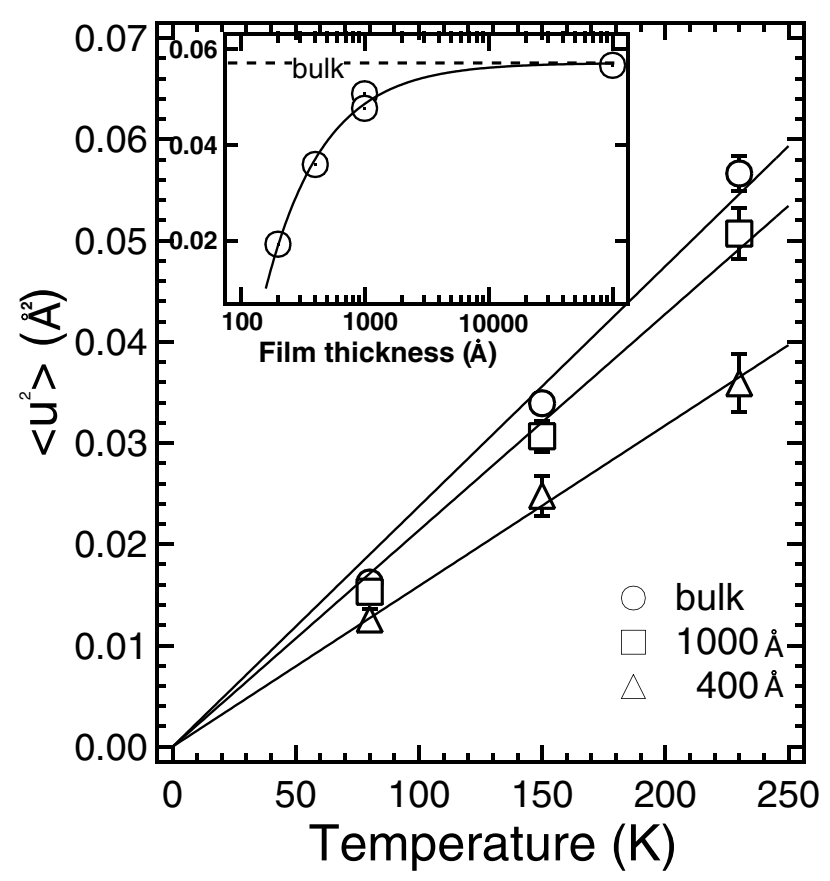

FIG. 2. Temperature dependence of mean square displacement $\left\langle u^{2}\right\rangle$ : bulk $(\bigcirc), 1000 \AA(\square)$, and $400 \AA(\triangle)$. The inset shows the thickness dependence of $\left\langle u^{2}\right\rangle$ at $230 \mathrm{~K}$, and the solid curve is a guide to the eye.

possible explanation is as follows [16]. When polymer chains are confined in a film thinner than twice of radius of gyration $2 R_{g}$, polymer chains cannot sustain the normal coil conformation, and hence would be deformed. Such deformed polymer chains would experience higher restoring force than the normal coil (confinement induced hardening), so that the higher $f$ was observed with decreasing film thickness. The radius of gyration $R_{g}$ of this polymer under the Gaussian coil approximation is $\sim 150 \AA$ [17] and $2 R_{g}$ is $\sim 300 \AA$. The film thickness is almost comparable to $2 R_{g}$ in the case of $400 \AA$, and hence the above idea may be applicable to the reduction in $\left\langle u^{2}\right\rangle$ or to the increase in $f$ with decreasing film thickness. However, this idea may not hold for the case of $1000 \AA$ films, because the film is much thicker than $2 R_{g}$. Another possibility may be considered for this result. A hard (high $f$ ) dead layer near the interface is one of the possibilities which has been employed to explain the reduction in the thermal expansibility [18]. The thickness of the dead layer was estimated to be $55 \pm$ $5.0 \AA$ ̊ [18] and $37 \pm 10 \AA$ [19]. Assuming that a dead layer $50 \AA$ thick is independent of the total thickness, for example, the force constant of the dead layer $f_{\text {dead }}$ can be evaluated to be $24.4 \pm 4.6 \mathrm{~N} / \mathrm{m}$, which is about 4 times larger than that of bulk, and the difference between the bulk and the $1000 \AA$ thin films would be observed. At the moment, however, it is hard to distinguish which factor is dominant, especially for the films thinner than $2 R_{g}$. In any case, the mean square displacement $\left\langle u^{2}\right\rangle$ decreases or the 
corresponding force constant $f$ increases with decreasing the film thickness.

Next we focus on the inelastic and/or quasielastic scattering. The observed $S(Q, \omega)$ at $80 \mathrm{~K}$ was converted to the density of phonon states $G(\omega)$ after subtracting the elastic contribution and correcting for the Debye-Waller factor. The results are shown in Fig. 3 for the bulk and the thin films 1000 and $400 \AA$ thick. It is clear that the density of states $G(\omega)$ decreases with the film thickness, agreeing with the reduction in $\left\langle u^{2}\right\rangle$ observed in the elastic scattering. In fact, the mean square displacement $\left\langle u^{2}\right\rangle$ calculated from the observed $G(\omega)$ agrees with the observed one [20], showing that the decrease in $\left\langle u^{2}\right\rangle$ is caused by the reduction of $G(\omega)$. In the energy region of this experiment between 0.20 and $10 \mathrm{meV}$, there exist at least two modes [21]: the localized boson peak mode and the Debye mode (or the sound wave). In soft materials or at high temperatures, additional relaxation modes are often observed even in the glassy state [22]. Here we consider to which mode the reduction in $G(\omega)$ is attributable.

As seen in Fig. 3, the boson peak energy (or frequency) is almost unaltered with film thickness, at least within the experimental error. In porous media, it is also reported that the boson peak energy of dibutyl phthalate containing $5 \mathrm{~mol} \%$ ferrocene is not changed [23]. These results suggest that the hardening of the boson peak mode does not occur in confinement. Nevertheless, we observed the reduction in $G(\omega)$ with the film thickness, implying that the hardening may occur in the Debye mode. Assuming that the amplitude of the boson peak mode is the same as the Debye mode, the Debye contribution to $G(\omega)$ was calculated for the bulk and the thin films (Fig. 3). $G_{D}(\omega)$ of the Debye mode is related to the average sound velocity $\nu$ through $G_{D}(\omega)=\frac{4 \pi V \omega^{2}}{\nu^{3}}$, where $V$ is the average atomic volume, and hence the reduction in $G_{D}(\omega)$ corresponds to the increase in the sound velocity caused by the potential

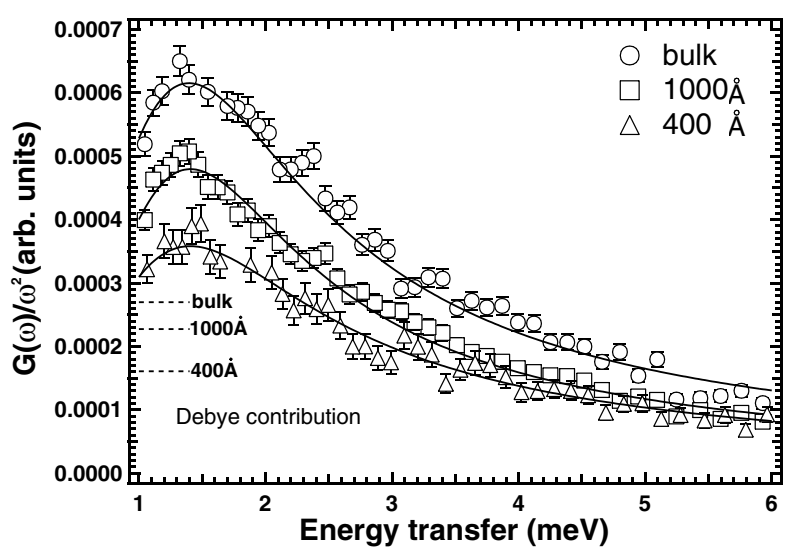

FIG. 3. Density of states $G(\omega)$ calculated after subtracting the elastic component and correcting for the Debye-Waller factor: bulk $(\bigcirc), 1000 \AA(\square)$, and $400 \AA(\triangle)$, and the solid curve is a guide to the eye. Dashed lines show the Debye contributions. hardening due to the spatial confinement of polymer chains and/or the existence of the dead layer. If the boson peak modes are just reduced and the high frequency mode out of the present measurement ( $>10 \mathrm{meV}$ ) increases, the average force constant would increase. This is another possibility.

We also briefly discuss the cutoff problem of the long wavelength phonon due to the limited scale [24]. The cutoff energy (or frequency) for the $400 \AA$ PS thin film was calculated to be about $0.17 \mathrm{meV}$ or less. Note that this is the maximum cutoff energy because of the anisotropic confinement in the thin film. This energy is located within the elastic scattering in this experiment $(\delta E=0.2 \mathrm{meV})$, so that the cutoff of the long wavelength phonon is not the cause for the reduction in the inelastic intensity above $0.2 \mathrm{meV}$.

The observed dynamic scattering law $S(Q, \omega)$ was scaled by the Bose factor after the correction for the Debye-Waller factor using $\left\langle u^{2}\right\rangle$ evaluated from the elastic scattering. The scaled spectra are shown in Fig. 4 for the bulk and the 1000 and $400 \AA$ films, where the reference temperature is $80 \mathrm{~K}$. The spectra were well scaled in the energy range above about $1.5 \mathrm{meV}$ including the boson peak, while below $1.5 \mathrm{meV}$ the excess scattering was slightly observed. This indicates the appearance of the quasielasticlike picosecond fast relaxation process $[25,26]$. By subtracting the Bose-scaled component we could estimate the quasielastic scattering component. We integrated the spectrum in the quasielastic region ( 0.39 to $1.5 \mathrm{meV}$ ) and plotted the integrated intensity against temperature in Fig. 5. The integrated intensities increase with temperature for the bulk and the $1000 \AA$ film, while it is almost independent of temperature for the $400 \AA$ film, showing that the fast process is depressed in the $400 \AA$ film. This tendency is clearly observed above the glass transition temperature [27]. The fast process in bulk PS has been assigned to a motion within a potential well (cage

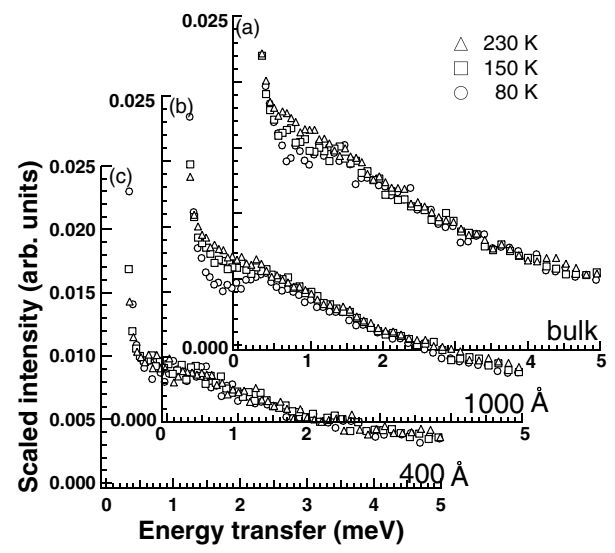

FIG. 4. Bose-scaled inelastic scattering intensity after correction for the Debye-Waller factor using $\left\langle u^{2}\right\rangle$ evaluated from elastic scattering: (a) bulk, (b) $1000 \AA$, and (c) $400 \AA$. The reference temperature is $80 \mathrm{~K}$. 


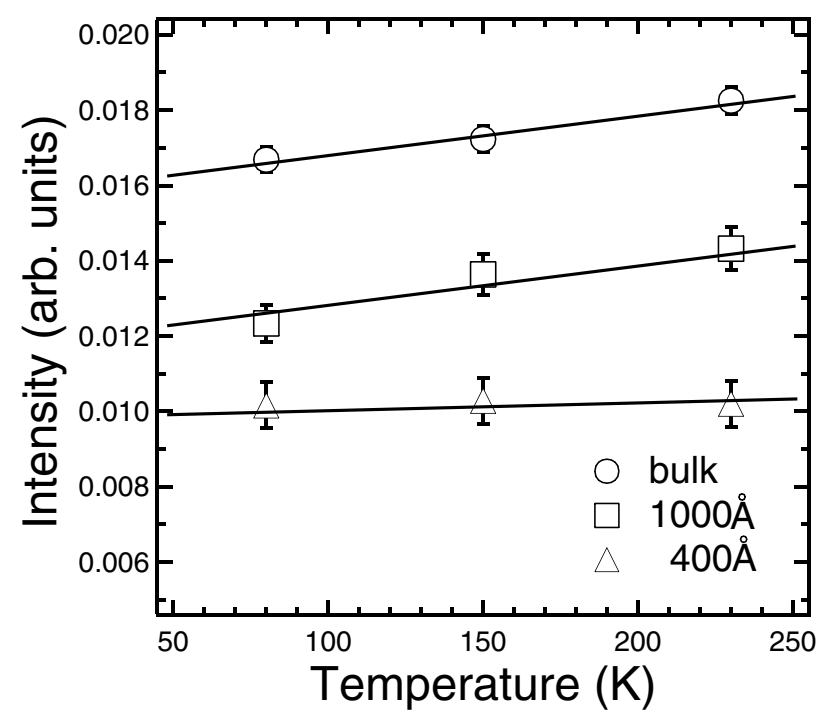

FIG. 5. Temperature dependence of integrated intensity in the quasielastic region (0.39 to $1.5 \mathrm{meV})$ as a function of temperature: bulk $(\bigcirc), 1000 \AA(\square)$, and $400 \AA(\triangle)$.

motion) $[25,26]$. If the force constant related to the fast process is hardened, the amplitude decreases and the characteristic time might increase, leading to the reduction in $\left\langle u^{2}\right\rangle$. It should be noted that the fraction of the fast process in $S(Q, \omega)$ is not large in the low temperature range far below $T_{g}$, and hence the reduction in $\left\langle u^{2}\right\rangle$ is mainly due to the hardening of the Debye mode owing to the spatial confinement of polymer chains and/or the existence of the dead layer.

In this study, the elastic, inelastic, and quasielastic neutron scattering results have demonstrated that the potential hardening occurs in the thin films in the meV region, especially in the Debye mode. This has been assigned to the effects of the spatial confinement of polymer chains and/or the interfacial hard dead layer. In the thin films, anisotropic motions are easily expected; however, the present results are on the average of the motional directions. This problem is now under investigation.

*Corresponding author.

Electronic address: kanaya@scl.kyoto-u.ac.jp

[1] Polymer Surfaces, From Physics to Technology, edited by F. Garbassi, M. Morra, and E. Occhiello (Wiley, Chichester, 1998).
[2] Polymer Surface, Interfaces and Thin Films, edited by A. Karim and S. Kumar (World Scientific, Singapore, 2000).

[3] J. L. Keddie, R. A. L. Jones, and R. A. Cory, Europhys. Lett. 27, 59 (1994).

[4] J. L. Keddie, R. A. L. Jones, and R. A. Cory, Faraday Discuss. 98, 219 (1994).

[5] S. Kawana and R. A. L. Jones, Phys. Rev. E 63, 21501 (2001).

[6] J. A. Forrest, K. Dalnoki-Veress, J. R. Stevens, and J. R. Dutcher, Phys. Rev. Lett. 77, 2002 (1996).

[7] J. A. Forrest, K. Dalnoki-Veress, and J. R. Dutcher, Phys. Rev. E 56, 5705 (1997).

[8] W. J. Orts, J. H. v. Zanten, W. Wu, and S. K. Satija, Phys. Rev. Lett. 71, 867 (1993).

[9] J. A. Forrest and R. A. L. Jones, in Ref. [2], p. 251.

[10] T. Miyazaki, K. Nishida, and T. Kanaya, Phys. Rev. E 69, 022801 (2004); 69, 061803 (2004).

[11] C. L. Soles, J. F. Douglas, W. Wu, and R. M. Dimeo, Phys. Rev. Lett. 88, 037401 (2002).

[12] B. Frick, K. Dalenoki-Veress, J. A. Forrest, J. Dutcher, C. Murray, and A. Higgins, Eur. Phys. J. E 12, 93 (2003).

[13] F. Y. Hansen, L. Criswell, D. Fuhrmann, K. W. Herwig, A. Diama, R. M. Dimeo, D. A. Neumann, U. G. Volkmann, and H. Taub, Phys. Rev. Lett. 88, 037401 (2002).

[14] R. Inoue and T. Kanaya (unpublished).

[15] S. Rastogi and V. D. Gupta, J. Macromol. Sci. Phys. B 34, 1 (1959).

[16] T. Kanaya, T. Miyazaki, R. Inoue, and K. Nishida, Phys. Status Solidi (b) 242, 595 (2005).

[17] J. Brandrup, E. H. Immergut, and E. A. Grulke, Polymer Handbook (John Wiley \& Sons, New York, 1998).

[18] G. B. DeMaggio, W.E. Frieze, D. W. Gidley, M. Zhu, H. A. Hristov, and A.F. Yee, Phys. Rev. Lett. 78, 1524 (1997).

[19] K. Fukao and Y. Miyamoto, Phys. Rev. E 61, 1743 (2000).

[20] In the calculation the absolute value of $G(\omega)$ was obtained by comparing the present data with the absolute data previously measured by IN6, ILL.

[21] K. Inoue, T. Kanaya, S. Ikeda, K. Kaji, K. Shibata, M. Misawa, and Y. Kiyanagi, J. Chem. Phys. 95, 5332 (1991).

[22] U. Buchenau, C. Schönfeld, D. Richter, T. Kanaya, K. Kaji, and R. Wehrmann, Phys. Rev. Lett. 73, 2344 (1994).

[23] T. Asthalter, M. Bauer, U. van Bürck, I. Sergueev, H. Franz, and A. I. Chumakov, Eur. Phys. J. E 12, 9 (2003).

[24] R. Zorn, L. Hartmann, B. Frick, D. Richter, and F. Kremer, J. Non-Cryst. Solids 307-310, 547 (2002).

[25] T. Kanaya, T. Kawaguchi, and K. Kaji, J. Chem. Phys. 98, 8262 (1993).

[26] T. Kanaya, T. Kawaguchi, and K. Kaji, J. Chem. Phys. 104, 3841 (1996).

[27] R. Inoue and T. Kanaya (unpublished). 